\title{
DIFFERENTIAL EFFECTS OF INTERLEUKIN 6 AND LIPOPOLYSACCHARIDE ON TELEOST B LYMPHOCYTES
}

\author{
Beatriz Abós ${ }^{1}$, Tiehui Wang ${ }^{2}$, Rosario Castro ${ }^{1}$, Esther Leal ${ }^{1}$, Alfonso Luque ${ }^{1}$, Jeffrey \\ Havixbeck $^{3}$, Daniel Barreda ${ }^{3}$, Chris Secombes ${ }^{2}$ Carolina Tafalla $^{1}$. \\ ${ }^{I}$ Centro de Investigación en Sanidad Animal (CISA-INIA), Madrid, Spain. \\ ${ }^{2}$ Scottish Fish Immunology Research Centre, University of Aberdeen, UK \\ ${ }^{3}$ Department of Biological Sciences, University of Alberta, Alberta, Canada
}

In mammals, interleukin-6 (IL-6) is a pleiotropic cytokine that has the capacity to induce the differentiation of B cells into antibody-secreting cells (ASCs). On the other hand, lipopolysaccharide (LPS) acts on B cells as a $\mathrm{T}$ cell independent antigen producing a polyclonal cell activation that goes along with an increased proliferation and antibody secretion. LPS is also known to enhance the B cell antigen-presenting capacity of B cells. Very few studies have examined how teleost B cells respond to these two stimuli, thus the aim of our work was to evaluate the stimulatory capacity of recombinant rainbow trout (Oncorhynchus mykiss) IL-6 on spleen B cells, comparing its effects to those induced by LPS. We found that both IL- 6 and LPS induced the proliferation of spleen $\operatorname{IgM}^{+}$B cells and activated $\mathrm{NF}-\kappa \mathrm{B}$ in these cells as determined by NF- $\mathrm{BB}$ translocation to the nucleus. Additionally, IL-6 and LPSstimulated B cells mobilized more intracellular calcium after B cell receptor cross-linking. Although both IL-6 and LPS provoked a significant differentiation of B cells to ASCs as determined by ELISPOT, the levels of secreted IgM mRNA only increased in response to IL-6, suggesting different pathways for increased antibody secretion. Concerning effects on their antigen-presenting capacities, IL-6 and LPS showed quite different effects. We observed a significant down-regulation of MHC-II surface expression on IL-6-stimulated B cells, but a significant up-regulation with LPS in comparison with control cells. Accordingly, we found a significant increase of the percentage of phagocytic $\operatorname{IgM}^{+} \mathrm{B}$ cells when splenocytes were stimulated with LPS but not with IL-6. This work provides important insights on the B-stimulating capacities of IL-6 and LPS not previously explored in teleost fish.

\section{KEYWORDS}

B cells; Interleukin 6 (IL-6); lipopolysaccharide (LPS); trout

§ Corresponding author. Tel.+34 916202300; Fax +34 916202247.

Email address: tafalla@inia.es 